\title{
LA CORRESPONDANCE DE JEAN CHAPELAIN : TÉMOIGNAGE D'UNE AMITIÉ SINCÈRE ET UTILITAIRE ENTRE HOMMES DE LETTRES
}

Jean Chapelain échange des lettres avec un nombre de correspondants qui va croissant au fur et à mesure que grandit sa notoriété auprès des autorités de son époque. Il échange de la correspondance surtout avec des hommes de lettres (Guez de Balzac, Pierre Corneille, Georges de Scudéry), des ministres et de puissants aristocrates (Colbert, le duc de Montausier ou le duc de Longueville), parfois des femmes (Mlle de Gournay, Mlle de Scudéry, Mme de Sévigné) et des érudits étrangers (Nicolas Heinsius, Carlo Dati, Christian Huygens), pour n'en citer que quelques-uns.

Les relations sociales se fondent sur des intérêts communs ; celles de Chapelain se nouent surtout au sein de ce qu'on peut appeler la « République des lettres ». Les humanistes du siècle précédent ont peut-être servi d'exemple au milieu littéraire que fréquente Chapelain, car ils « ont pratiqué l'amitié de manière ostentatoire. Les dédicaces, les recueils de lettres, les échanges de portraits et de cadeaux, l'hospitalité : tout était porté à la connaissance du « public»—c'està-dire le groupe dans lequel se meut le réseau et qui constitue son horizon de référence ${ }^{1}{ }^{1}$. Certes, Chapelain et ses correspondants se manifestent leur amitié de façon semblable, mais ils forment un réseau d'échanges épistolaires et non pas un réseau social ${ }^{2}$. Chapelain fait partie de plusieurs cercles : institutionnalisé

${ }^{1}$ M. Daumas, Des trésors d'amitié. De la Renaissance aux Lumières, Armand Colin, Paris 2011, p. 170.

${ }^{2}$ Ch. Jouhaud, « Sur le statut d'homme de lettres au XVII ${ }^{\mathrm{e}}$ siècle. La correspondance de Jean Chapelain (1595-1674) », Annales. Histoire, Sciences Sociales, 49e année, N 2, 1994, pp. 312 et 
(l'Académie française), savant (le cabinet Dupuy) et mondain (le salon de Mme de Rambouillet) et cela, forcément, élargit son pôle de connaissances et ne limite pas ses centres d'intérêts aux seules dimensions politiques ou matérielles (avantages financiers, publications, etc.). Il a donc des correspondants officiels, tel Colbert qui le fait travailler pour glorifier le règne de Louis XIV ou des érudits que Chapelain sollicite à son tour pour qu'ils rendent hommage au roi, et des correspondants privés, au large sens du terme, qui appartiennent à la famille du marquis de La Trousse, au milieu mondain et savant de la capitale.

Conscient du caractère à la fois officiel et privé de ses missives, il les gardait précieusement et s'est soucié de ce qu'il en adviendrait même après sa mort. Dans son testament, il précise que toutes les lettres (les siennes et celles de ses correspondants) doivent rester dans leur coffre et ne pourront être publiées que pour défendre sa réputation ${ }^{3}$. Les dernières volontés de Chapelain n'ayant pas été respectées, ses lettres donnent une image de la vie intellectuelle des années trente à soixante-dix de son siècle. Sa riche correspondance permet de voir en lui non seulement un savant critique qui, de son vivant, jouissait d'un grand prestige et fut à la fois victime d'une haine moqueuse, mais révèle aussi un être humain. Un ami.

«La liberté de laquelle nous usons ensemble est la plus chère production de notre amitié. C'est le fruit du mariage de nos esprits et le gage de notre fidélité mutuelle. Je l'entretiendrai toujours comme la chose qui m'est la plus précieuse et par laquelle le monde ne m'est pas tout à fait odieux $»^{4}(28 \mathrm{X} 1632 ; \mathrm{I}, 7)$, écrit-il à Godeau, caractérisant bien la nature de l'amitié qui a pour principe la réciprocité, la communauté d'esprits, la fidélité, la franchise et donne un sens à la vie. Ajoutons à cette caractéristique le désir d'une amitié éternelle et l'image de l'ami qui est un autre soi-même : «Entre les choses qui me seront les plus dures et les plus amères quand la trompette sonnera et qu'il faudra partir, vous ne doutez point que ce ne soit notre séparation puisque je vous considère comme un autre moi-même et que je ne puis rien laisser qui me touche de plus près $»^{5}$, déclare-t-il à Nicolas Heinsius. Une amitié idéalisée, conforme au principe de la philia humaniste, ne cesse de se manifester dans les lettres à ses amis hommes de lettres.

$331 ;<$ www.persee.fr/doc/ahess_0395-2649_1994_num_49_2_279263> [consulté le 15 septembre 2016].

${ }^{3}$ G. Collas, Un poète protecteur des lettres au XVII s. Jean Chapelain 1595-1674, Paris 1912 ; Slatkine Reprints, Genève 1970, p. 473.

${ }^{4}$ Lettres de Jean Chapelain de l'Académie Française, par $\mathrm{Ph}$. Tamizey de Larroque, M DCCC LXXX, coll. Documents inédits sur l'histoire de France, Imprimerie Nationale, Paris 1968, II vol. : t. 1 (septembre 1632-décembre 1640), t. 2 (2 janvier 1660-20 décembre 1672). Toutes les citations des lettres de Chapelain (sauf celles à N. Heinsius) proviennent de cette édition et sont suivies entre parenthèses de la date (si elle n'est pas donnée dans le texte), du numéro du volume et de la page. L'orthographe a été modifiée pour la clarté du texte.

5 J. Chapelain, Les lettres authentiques à Nicolas Heinsius (1649-1672). Une amitié érudite entre France et Hollande, édition établie, introduite et annotée par B. Bray, Honoré Champion, Paris 2005 , p. 258. 
Toute conforme qu'elle soit à cet ancien principe, l'amitié aux temps de Chapelain ne doit pas être forcément réciproque et signifie aussi l'utilité. Un bref parcours des notices de dictionnaires permet de s'en rendre compte : le dictionnaire de Richelet (1679) présente la réciprocité comme un trait indispensable de l'amitié : " affection réciproque qu'on se témoigne pour de particulières considérations ». Ce même dictionnaire atteste que ce « qui peut faire naître l'amitié, c'est d'obliger, de faire du bien $»^{6}$. Furetière (1689) affirme au contraire que c'est « une affection qu'on a pour quelqu'un, soit qu'elle soit seulement d'un côté soit qu'elle soit réciproque », mais continue de parler de l'utilité : « [l'amitié] signifie encore plaisirs, bons offices $»^{7}$. Pour le dictionnaire de l'Académie Française (1694), l'amitié n'implique pas forcément la réciprocité : c'est une «affection mutuelle, réciproque entre deux personnes à peu près de l'égale condition $[\ldots]$ mais se dit aussi quoique l'affection ne soit pas réciproque $»^{8}$. L'aspect utilitaire disparaît tandis qu'on souligne l'appartenance au même groupe social, exigence qui correspond au système fortement hiérarchisé de l'époque.

Réciproque ou pas, l'amitié résulte d'un choix et « constitue un acte de pouvoir, elle fait d'autrui un obligé ${ }^{9}$. Ce dernier aspect, fondement de l'amitié utilitaire, est évoqué sans détour par La Rochefoucauld qui n'hésite pas à priver le lecteur de toute idéalisation des motivations humaines : « Ce que les hommes ont nommé amitié n'est qu'une société, qu'un ménagement réciproque d'intérêts, et qu'un échange de bons offices ; ce n'est enfin qu'un commerce où l'amour-propre se propose toujours quelque chose à gagner $»^{10}$. Certes, c'est sur «l'échange de bons offices » que se fondent les relations de Chapelain avec ses correspondants. L'utilité exclut-elle pour autant un attachement, une fraternité à laquelle les intérêts communs viennent s'ajouter ou dont ils résultent? Peut-on distinguer les vrais amis des correspondants liés à Chapelain par pur intérêt? Nous allons examiner ses lettres à ses amis écrivains pour voir quelle place il y réserve à l'amitié.

«Le temps qui fortifie l'amitié affaiblit l'amour » affirme La Bruyère ${ }^{11}$. Si on examine la correspondance de Chapelain, le critère de la durée, sans être unique et décisif, confirme sa fidélité à certains de ses amis : sa correspondance avec Guez de Balzac a duré plus de vingt ans avant d'être interrompue par la mort de son ami en 1654, et celle avec Nicolas Heinsius, vingt-quatre ans, après quoi les deux amis

${ }^{6}$ P. Richelet, Dictionnaire françois..., <http://catalogue.bnf.fr/ark:/12148/cb351540398> [consulté le 10 septembre 2016].

${ }^{7}$ A. Furetière, Dictionnaire universel..., <http://catalogue.bnf.fr/ark:/12148/cb30470957z> [consulté le 10 septembre 2016].

${ }^{8}$ Dictionnaire de l'Académie française, $<$ http://catalogue.bnf.fr/ark:/12148/cb35153876f > [consulté le 10 septembre 2016].

${ }^{9}$ M. Daumas, op. cit., p. 13.

${ }^{10}$ La Rochefoucauld, Réflexions ou Sentences et Maximes morales, J. Lafond (éd.), Folio classique, Paris 1976, maxime 473.

${ }^{11}$ La Bruyère, Les Caractères ou les mours de ce siècle, R. Pignarre (éd.), Garnier Flammarion, Paris $1965(\mathrm{IV}, 3)$. 
ont été séparés par la guerre de Hollande ${ }^{12}$, deux ans avant la mort de Chapelain en 1674. La force de l'attachement amical ne faiblit pas : plusieurs années après la mort de Balzac, Chapelain évoque leurs liens «si vieux et si chers qu'ils ne sauraient manquer d'être indissolubles » et son rôle auprès de Balzac dont il a été « directeur et réviseur de ses ouvrages » et « son ami à toute épreuve » (à M. Spanheim, 21 XII 1659; II, 71-72). À son tour, l'archidiacre d'Angoulême, héritier des documents de Balzac, parle de la publication de ses lettres à Chapelain comme d'une " marque de l'estime » que Balzac portait à l'amitié et à la vertu de son $\mathrm{ami}^{13}$. Quand on examine la fréquence des échanges, ceux avec Balzac dépassent d'ailleurs largement le nombre de lettres envoyées aux autres correspondants pendant la même période ${ }^{14}$.

Parmi ses amis français, Balzac tient une place privilégiée ${ }^{15}$ et parmi les érudits étrangers, c'est Heinsius, ami hollandais, qui a en quelque sorte pris la relève de Balzac après sa mort ${ }^{16}$. Il y en eut aussi d'autres avec qui Chapelain était très lié, tels Valentin Conrart, Pierre-Daniel Huet, Antoine Godeau, François Le Métel de Boisrobert, Pierre Gassendi, Claude Vaugelas ou encore Gilles Ménage, cet ami-ennemi avec qui la relation a été tumultueuse.

Tout au long de sa correspondance, Chapelain est lié avec ses amis écrivains par une coopération littéraire, un échange mutuel de jugements et de compliments :

Vous ne ferez jamais rien de moi qu'en me châtiant et qu'en m'ouvrant les yeux sur les défauts dont toutes mes productions sont remplies. C'est une grâce que j'attends de vous, laquelle si je n'eusse pas cru l'obtenir, je ne me fusse jamais résolu à vous obéir en ce qui regarde la révision de vos ouvrages, la connaissant infiniment au-dessus de tout ce que je pourrai jamais (à Balzac, 25 IX 1632 ; I, 3).

La réciprocité de la confiance dans le jugement de l'autre implique la supériorité de l'ami, idée qui revient fréquemment dans les lettres (surtout avec Balzac) et constitue un compliment que la bienséance impose, mais que l'amitié n'exclut pas.

${ }^{12}$ Le 18 février 1672, Chapelain prévoit les difficultés à venir : «Comme je vois de toutes parts les choses disposées ce pourra bien être la dernière que vous recevrez de nous pour ne pas nuire auprès de vos Gouverneurs qui sont une Nation fort soupçonneuse bien que notre commerce ne soit que de l'intérêt des Muses et qu'il n'y ait rien de plus innocent » (J. Chapelain, Les lettres authentiques à Nicolas Heinsius ..., p. 565).

13 Épître à M. le duc de Montausier, [dans :] Lettres familières de M. Balzac à M. Chapelain, chez Louis et Daniel Elzevier, Amsterdam 1661.

${ }^{14}$ Entre 1632 et 1640, Balzac a reçu 118 lettres de Chapelain tandis que le suivant sur la liste, le duc de Montausier, en a reçu 97 (cf. Ch. Jouhaud, op. cit., p. 332).

15 G. Collas, op. cit., p. 293.

${ }^{16}$ Sur N. Heinsius, $c f$. éd. B. Bray, introduction à son édition des lettres de Chapelain (J. Chapelain, Les lettres authentiques à Nicolas Heinsius..., pp. 13-28) ; M. Kulesza, «Quand les érudits sont amis : exemple de la correspondance de Jean Chapelain avec Nicolas Heinsius », [dans :] L'amitié dans les écrits du fort privé et les correspondances du Moyen-Âge à 1914, actes réunis et présentés par M. Daumas, PUPPA, Pau 2014, pp. 97-109. 
Il est en effet difficile de différencier ce qui est conventionnel de ce qui est sincère dans les expressions affectives. Chapelain utilise parfois des tournures chaleureuses et recherchées ${ }^{17}$, mais son vocabulaire émotionnel (même si aujourd'hui il paraît excessif) ne diffère pas beaucoup des formules de politesse et déclarations de sentiments pratiquées dans les salons. Les lettres à Balzac et à Heinsius ${ }^{18}$, surtout, diffèrent cependant des autres. Il ne s'agit pas tant des formules utilisées que du ton de sincérité et d'exclusivité qui est réservée à chacun d'eux.

Chapelain écrit des lettres dont il sait qu'elles seront lues ou copiées. Il lui arrive d'ajouter la lettre de quelqu'un au paquet qu'il envoie à un correspondant commun. Mais dans une lettre à Balzac, il souligne le caractère privé et exclusif de leur échange :

Ne serait-ce pas une chose bien rude pour le nom que votre amitié et vos éloges m'ont donné, que l'on vît, après moi, ce grand nombre d'embryons et d'imaginations indigestes qui ont été conçues et enfantées en même temps pour servir en une seule occasion et une seule fois entre deux personnes seules, et seulement pour ce que je n'ai pas la voix si forte qu'on la puisse entendre d'ici en Angoumois au-delà les monts (23 II 1639; I, 392).

Réviser les ouvrages les uns des autres, se juger mutuellement, s'entraider était habituel dans le groupe d'intellectuels et Chapelain était le premier à le faire. Ce passage insistant sur l'exclusivité ne surprend pas uniquement si on songe au caractère exceptionnel de la relation amicale avec Balzac.

Pourtant, entre les deux écrivains, la part de la franchise du jugement et la part réservée à la bienveillance que commande l'amitié posent parfois un problème. L'amitié doit aussi savoir s'expliquer :

Quand vous ne seriez pas le grand homme que vous êtes, il aurait suffi de mon affection pour me rendre en vous toutes choses agréables. Vous m'avez toujours plu et en tout, mais j'ai eu sujet de vous dire par les discours de ceux qui n'ont pas pour vous la passion que j'ai, que vous n'aviez pas plu à tout le monde, comme vous le méritez et comme je le désire (7 II 1633 ; I, 26).

Faut-il rapporter à un ami tout ce qu'on dit de lui ? Chapelain explique « ses scrupules » : d'un côté le devoir de l'amitié consiste à ne rien cacher à son ami, mais si cela lui déplaît (comme c'est le cas de Balzac), il faut accepter la volonté de l'ami, sans renoncer à le convaincre de ses raisons car il s'agit de son bien :

[...] je vais me décharger de ce soin assidu que je m'étais prescrit sur l'opinion que vous l'exigiez de ma sincérité. Quoique je les [les scrupules] estime de quelque mérite, je ne les puis néanmoins aimer, s'ils sont capables de vous altérer contre moi, et pourvu que vous ne m'en imputiez pas la négligence, je m'en abstiendrai bien plus volontiers que je ne les pratiquerai. Je vous dis ceci avec

17 Citons juste un exemple : «je ressens pour vous les inquiétudes des amants et je reconnais encore en cela que je vous aime bien fort » (à Boisrobert, [en blanc] XI 1633 ; I, 55).

${ }^{18}$ B. Bray souligne la spontanéité du langage d'amitié de Chapelain dans les lettres à Heinsius : « On ne peut pas négliger ces déclarations, les taxer de mensongères ou les considérer comme de simples ornementations du discours épistolaire. Ce langage n'est pas habituel dans la correspondance de Chapelain » (cf. éd. B. Bray, op. cit., p. 24). 
ma candeur ordinaire et vous prie de croire que je suis et serai toujours ce même ardent ami que vous m'avez cru jusqu'ici, sans que ce nouvel ordre diminue en rien le véhément désir que j'ai de votre avantage et de votre gloire (7 II 1633 ; I, 26).

Balzac était un homme susceptible et seul un véritable ami trouvait des mots suffisamment doux, chaleureux et en même temps fermes pour amadouer le mécontent et sauver l'amitié.

Après le départ de Balzac à Angoulême, Chapelain assure le lien entre l'exilé et le milieu parisien, l'Hôtel de Rambouillet en l'occurrence. Il le sollicite et l'encourage à prendre part aux querelles littéraires qui animent le salon (celle des Supposés ${ }^{19}$ ou la querelle du $\mathrm{Cid}^{20}$ ), ce qui a un effet thérapeutique sur l'exilé sombrant de plus en plus dans la solitude. Chapelain en est conscient et joue souvent ce rôle auprès de Balzac. Plusieurs fois il l'assure qu'il comprend son désir de solitude, opposé à sa propre vie parisienne qui lui pèse parfois. Se plaindre et se soutenir mutuellement est un rôle d'ami dévoué. Ainsi le 27 novembre 1633, Chapelain fait part à Balzac de ses sentiments sur son propre mode de vie, différent de celui de son ami qui sait stoïquement supporter « les entraves de la fortune» :

J'aurais un long discours à vous faire si je vous voulais exprimer mes sentiments sur ma fortune et surtout combien je suis peu d'accord avec ceux qui m'estiment heureux, parce qu'ils me voient chargé de chaînes d'or. [...] Dans ma servitude je vous considère comme un Souverain et ne vous puis assez louer de vous être affranchi glorieusement des entraves de la fortune. Je suis marri que mes fers soient illustres et que je ne les puisse quitter sans être blâmé. [...] Je ne suis plus homme, je suis Courtisan (I, 57-58).

Ce passage témoigne du caractère privé et sincère des lettres à Balzac, car un homme aussi puissant que Chapelain, aussi bien coté auprès des autorités, ne dévoile pas ses doutes au grand jour. Et même s'il y a peut-être une sorte de coquetterie dans ses paroles, il n'y a pas de raisons de douter de sa franchise.

Quelques années après, la solitude de Balzac prend une dimension inquiétante car il s'isole de plus en plus et doute du sens de l'écriture. Le vigilant Chapelain est là pour le mettre en garde contre ce mode de vie. Comme dans d'autres lettres, dans celle-ci aussi, il comprend bien qu'il faut choisir soigneusement ses correspondants (cela est d'ailleurs valable et pour Balzac, et pour lui-même, tous les deux étant trop sollicités), mais il ne faut pas se couper du monde et refuser l'amitié. Dans cet exposé, il mêle la compréhension, l'inquiétude, et tout en évoquant la nécessité de la vie sociale, il explique de façon quasi pascalienne le sens de l'écriture :

$\mathrm{Au}$ reste, il serait bien malaisé, quelque protestation publique que vous puissiez faire de ne vouloir point de commerce avec les écrivains, que vous vous en puissiez défendre, à moins que de faire de

${ }^{19}$ La querelle entre Chapelain et Voiture au sujet des Supposés de l'Arioste qui a eu lieu en 1639.

${ }^{20}$ Sur Les sentiments de l'Académie française sur la tragi-comédie du «Cid», cf. J. Chapelain, Opuscules critiques, A.C. Hunter (éd.), introduction, révision des textes et des notes par A. Duprat, Droz, Genève 2007, pp. 80-97. 
votre solitude un désert aussi sauvage et aussi inaccessible que celui de M le Maistre qui, depuis sa retraite du monde, n'a pas même permis à mon amitié d'y entrer. Ce sera bien assez que vous vous en déchargiez en partie, et que ces protestations vous servent à vous délivrer des ambitieux importuns qui n'ont rien à faire, et qui sont bien aises de pouvoir dire qu'ils ont grand communication avec vous. Pour vos amis je ne sais pas même s'il serait à propos de garder un si opiniâtre silence, puisque vous êtes homme et que vous êtes obligé à pratiquer avec eux ce devoir de la société. Et ne me demandez point à quoi bon toutes ces écritures ? Quand elles ne serviraient point à la gloire de celui qui les fait ni à la satisfaction de ceux qui les reçoivent, elles servent du moins à passer la vie dans l'action, qui est une des obligations de l'homme et pour laquelle seule la nature l'a fourni de tant de nobles instruments qui ne peuvent demeurer qu'avec crime sans être employés [...] (30 XII $1640 ;$ I, 737-738).

Chapelain est sans conteste un homme sociable. Il est cordial et serviable, mais adapte les services et les cadeaux suivant la qualité du demandeur. À son époque, le portrait constitue un gage d'amitié et possède une valeur matérielle non négligeable. Chapelain est sollicité par Georges de Scudéry et par Nicolas Heinsius qui souhaitent avoir son portrait. Le premier n'est pas son ami intime, même si leur correspondance est suivie et si, dans la querelle du Cid, Chapelain, bon gré mal gré, a soutenu la plupart des arguments de Scudéry contre la pièce de Corneille. Mais la demande lui paraît excessive, car le portrait est un objet précieux qu'on n'offre pas à tout le monde. De plus Scudéry souhaite avoir celui de Chapelain pour des raisons de prestige : il veut l'ajouter à ceux qu'il a déjà accrochés dans son cabinet. Chapelain manifeste son mécontentement : « Je ne sais ce qui m'a fait paraître de mauvaise humeur pour M. Scudéry, si ce n'est l'importunité qu'il m'a donnée sur le sujet de mon portrait, lequel il m'a certes demandé et redemandé d'une manière bizarre dont il m'eut bien obligé de se passer » (à Balzac, 11 IX 1639 ; I, 493). Dans la suite Chapelain parle sur un ton ironique de Scudéry qui ne lui demande pas son avis sur ses ouvrages « et ne croit pas en avoir besoin $»$. Raison de plus de ne pas lui offrir son portrait.

Il n'en va pas de même quand c'est Heinsius qui le lui demande. Chapelain ne répond pas « oui » tout de suite ${ }^{21}$, car le portrait inséré dans l'édition de La Pucelle lui semble suffisamment ressemblant, mais change d'avis rapidement en évoquant son affection pour son ami hollandais : «Si vous persévérez néanmoins à l'ordonner je ne préférerai aucune occupation étrangère à celle de me livrer au Peintre pour me faire tirer $»^{22}$. L'attachement amical s'exprime par un discours galant.

Chapelain sait choisir ses contacts et réserve à ses meilleurs amis les meilleurs services, par exemple introduire un ami dans le cercle d'un autre. C'est ainsi qu'il introduit Conrart auprès de Balzac, en dressant de lui le portrait exemplaire d'un ami dévoué :

[...] c'est un homme de bon cœur et de bon esprit, un ami chaud et adroit et qui va toujours au-devant des occasions de faire office à ceux à qui il a voué de l'affection, surtout jaloux de sa parole

${ }^{21}$ Faut-il y voir aussi une marque d'avarice de Chapelain ? Cf. Tallement de Réaux, Historiettes, $<$ http://catalogue.bnf.fr/ark:/12148/cb314302816> [consulté le 8 septembre 2016].

${ }^{22}$ Lettre à Heinsius, éd. B. Bray, op. cit., p. 468. 
et qui se tient mieux obligé par sa promesse que par tout ce que les Lois ont inventé de liens et de chaînes pour tenir les hommes dans le devoir. Je le connais de longue main pour tel, et s'il n'était pas solvable, je voudrais le cautionner de tout ce que j'ai de générosité et de franchise (8 XII 1632 ; I, 11).

Chapelain apprécie les belles qualités humaines. Selon lui l'utilité est liée à l'attachement amical et il la trouve indispensable dans ses propres amitiés.

Chapelain est un homme puissant. Avant que Colbert ne l'emploie, dans les années 1630, pour dresser la liste des auteurs à faire pensionner par Louis XIV, le chancelier Séguier avait demandé à Boisrobert et à Chapelain de choisir les lettrés méritant une pension. Ainsi Antoine Gombaud, dit le chevalier de Méré, Guillaume Colletet, Claude Vaugelas se voient pendant quelque temps pensionnés par le mécène.

Par ailleurs, dans les années 1630 et 1640 surtout, Chapelain est le conseiller attitré de presque tous les écrivains. Il est toujours prêt à rendre service à un confrère : une amitié utilitaire le lie avec la plupart de ses correspondants, mais il attend en retour la réciprocité des sentiments et des services, voire des conseils et du soutien. Ainsi il a été sollicité par Vaugelas au sujet de sa traduction de La vie d'Alexandre Le Grand de Quinte-Curce ${ }^{23}$ et de son œuvre majeure, Remarques sur la langue française (1647). Vaugelas évoque avec déférence l'aide de Chapelain et le considère comme « un des plus grands génies de notre langue et de notre poésie héroïque ${ }^{24}$. À la même époque, Chapelain a consulté Vaugelas sur une ode destinée au cardinal Richelieu. Il l'appelle « un grand auteur » et lui demande de juger son poème sans complaisance :

C'est pourquoi je vous conjure de me désabuser, si ce que vous avez écrit de celle-ci n'est qu'un effet de votre affection ou de votre courtoisie. Prenez soin de ma pudeur que déjà vous avez ébranlée et qui peut faire naufrage sur votre foi. [...] Relisez les Vers encore une fois pour l'amour de moi, et que je sache votre sentiment particulier sur ce qui vous y choquera. Je veux que vous me payiez le devoir que je vous rends par votre sévère censure et prétend que vous m'y traitiez en Ennemi (10 VII $1633 ;$ I, 41)

C'est au nom de l'amitié justement que Chapelain exige la sévérité du jugement, comme s'il ne prenait pas en compte les exigences de la bienséance et l'affection amicale qui interdit de blesser celui qu'on aime bien.

Chapelain avait besoin d'amis pour acquérir plus d'assurance. Il est étonnant de voir que celui qui a été une autorité pour les autres et qui passait pour expert dans le domaine de la littérature et de la langue était si peu sûr de lui. Ses publications montrent ses réticences et son manque d'assurance : la traduction de Guzman d'Alfarache qu'il a faite pour ses élèves, les enfants du marquis de La

${ }^{23}$ Vaugelas y a travaillé dans les années 1620, elle a été corrigée dans les années 1630 et 1640 pour finalement paraître en 1650 .

${ }^{24} \mathrm{C}$. Vaugelas, Remarques sur la langue française utiles à ceux qui veulent bien parler et bien écrire, t. 1, éd. Chassang, Paris1881, <http://gallica.bnf.fr/ark:/12148/bpt6k6385630w/f203.item. $\mathrm{r}=$ Chapelain $>$ [consulté le 7 septembre 2016]. 
Trousse, est anonyme car c'est lui qui en a décidé ainsi : « de peur d'en amoindrir le mérite, mon nom que vous vouliez que je misse en tête a été par moi laissé $»^{25}$. De même l'épître qui précède la traduction, est remplie d'explications et d'excuses qui doivent amadouer les critiques potentiels ${ }^{26}$. La préface de La Pucelle commence par ces mots timides : «Je fais si peu de fondement pour le bon succès de ce poème, sur l'impatience qu'on a témoigné de sa publication, que je considère un si grand honneur comme son plus grand désavantage $»^{27}$. Et Chapelain d'évoquer ceux qui attendent son ouvrage pour en redire et ceux qui ont de bonnes intentions, mais qui seront déçus.

Chapelain « redoutait la critique, qu'il essayait de prévenir en cherchant sur ce qu'il pensait publier les conseils d'amis de confiance, tels Balzac et Conrart $»^{28}$, affirme A.C. Hunter, qui ajoute que s'il a mis plus de vingt ans à publier la première partie de sa Pucelle, c'est que « les ébauches en traînèrent entre les mains des nombreux amis consultés, dont les sages conseils devaient le mettre à l'abri des assauts qu'il appréhendait à juste titre $»^{29}$. Ce manque d'assurance implique aussi la susceptibilité. Du coup, il n'est pas étonnant de voir Chapelain supporter très mal la critique. Il a publié peu et aurait peut-être dû se limiter à ses ouvrages théoriques au lieu de se lancer dans la rédaction du volumineux poème héroïque qui lui a valu les louanges de quelques amis, mais aussi la critique moqueuse de ses nombreux adversaires.

En effet La Pucelle a déplu. Parmi d'autres, Jean de La Mesnardière en a fait une critique à laquelle Chapelain a préparé une réponse par écrit. Mais avant de l'imprimer, il l'a soumise à ses amis et, probablement sur leurs conseils, il y a renoncé. Le texte de Chapelain ne s'est pas conservé, mais Les remarques de M. Conrart sur la réponse de $M$. Chapelain qui se trouvent à la fin des Observations sur le poème de la Pucelle de La Mesnardière ${ }^{30}$ prouvent que Conrart s'est prononcé au nom de tout un cercle qui a formulé des objections aussi bien sur le fond que sur la langue de ce texte. Les amis ont donc déconseillé à Chapelain la publication de sa réponse, et ils lui ont peut-être ainsi évité un persiflage supplémentaire.

L'amitié a aussi été parfois source de déception pour Chapelain. C'est la rupture avec Ménage qui a été la plus pénible. Sans entrer dans les détails de cet événement ${ }^{31}$, il faut rappeler que Ménage, se sentant offensé par Gilles Boileau, a exigé de Chapelain qu'il refuse son soutien à ce dernier qui espérait entrer à l'Académie française. Chapelain s'en plaint à Christian Huygens :

\footnotetext{
25 J. Chapelain, Opuscules ..., p. 163.

${ }^{26}$ Ibidem, pp. $163-172$ et $176-183$.

27 Ibidem, p. 355.

28 A.C. Hunter, Le lexique de la langue de Jean Chapelain, Droz, Genève 1967, p. 12.

${ }^{29}$ Ibidem, p. 13.

30 J. de La Mesnardière, Lettre du sr Du Rivage contenant quelques observations sur le poème épique et sur le poème de la Pucelle, Paris 1656, BnF, microfiche, MFICHE Y-90.

${ }^{31}$ Cf. G. Collas, op. cit., pp. 310-318.
} 
[...] voyant que je ne voulais pas servir d'instrument à sa fureur ni devenir ministre de sa cruauté contre un homme qui, dans sa poursuite, ne lui faisait pas le moindre tort du monde, il [Ménage] a eu le mauvais courage de rompre avec moi après une amitié de plus de vingt années que lui-même confesse lui avoir été utile et honorable par mille sortes d'offices ardents et cordiaux (9 IV 1659; II, 34).

De façon claire, Chapelain souligne de nouveau sa serviabilité et son affection qui toutes les deux ont constitué le fondement de son amitié avec Ménage. Et même s'il assure à Huygens ne pas « s'en émouvoir » (II, 35), son chagrin continue et s'exprime sur un ton offensé et déçu :

J'étais le seul qui le connaissais et qui l'aimait avec ses imperfections et le seul qui par amitié lui représentais en toutes les occasions importantes les fausses mesures qu'il prenait dans sa conduite. Les obligations qu'il m'a depuis tant d'années, de son propre aveu, me donnaient ce droit d'un consentement si général que c'était toujours à moi qu'on avait recours lorsqu'on lui voulait faire entendre raison dans ses escapades, ce que je faisais cordialement et paternellement pour son avantage (à Heinsius, 13 V 1659 ; II, 37).

Dans la même lettre, Chapelain accuse Ménage d'être orgueilleux, présomptueux, de vouloir passer pour son « maître et son tyran », en un mot de l'offenser et d'oublier toute reconnaissance pour lui avoir été très utile. Ménage devient alors « cette Personne » et Chapelain oppose l'amitié sincère qui le lie avec Heinsius à celle qui vient d'être rompue et qui est devenue inimitié : « Je suis au moins assuré que cette division [...] n'arrivera jamais entre moi et vous, parce que nous ne sommes fous ni l'un ni l'autre et que l'ombre même de la tyrannie et de l'injustice est bannie de notre amitié $»{ }^{32}$.

Plus l'amitié est déçue, plus les anciens amis s'offensent mutuellement, cherchent à se discréditer et interprètent tout au désavantage de l'autre. Ménage a écrit un méchant poème sur Chapelain ; celui-ci n'a pas été en reste et a composé une épigramme mordante contre lui ${ }^{33}$. Plusieurs tentatives de réconciliation ont échoué $^{34}$. Chapelain craignait que Ménage veuille rejeter ses torts sur lui,

faisant croire à un besoin que nous serions les offenseurs et les repentants, trop heureux qu'on nous voulût bien pardonner, et que nos crimes fussent abolis par une générale amnistie. Ces plans sont dignes de leur cervelle démontée et il n'y a pas lieu de craindre que ni vous ni moi donnions dans ce panneau, n'étant pas, grâce à Dieu, aussi écervelés qu'ils le sont (à Conrart, 28 IX 1666 ; II, 478).

La fermeté des résolutions, la dignité de son attitude s'opposent sous la plume de Chapelain à l'injustice de l'ennemi. Le ton des lettres évoquant Ménage est tout

${ }^{32}$ Lettre à Heinsius, éd. B. Bray, op. cit., p. 258.

${ }^{33}$ L'amoureux et docte Ménage,

S'il faut en croire son langage

Depuis vingt ans ne s'est miré,

Ne pouvant plus voir son visage

Si hâve et si défiguré $[\ldots]$

(cf. G. Collas, op. cit., p. 315)

${ }^{34}$ Une demi réconciliation n'aura lieu qu'en 1671 ( $c f$. la lettre à Heinsius, éd. B. Bray, op. cit., pp. 544-545). 
aussi émotionnel que celui dont il usait pour exprimer ses sentiments d'amitié à Balzac ou à Heinsius. L'amitié trahie éveille des émotions aussi fortes que l'amour déçu. De plus, quand l'amitié est rompue, le souvenir de services rendus dans le passé éveille l'accusation d'ingratitude qui s'ajoute à la déception affective. Ainsi le côté utilitaire de l'amitié est tout aussi fort que son aspect émotionnel, et on le voit le mieux lors de la rupture.

Les lettres de Chapelain à ses amis écrivains témoignent de son besoin absolu d'amitié. Cet homme, dont l'histoire a gardé l'image d'un docte persiflé, s'avère être un ami dévoué et sensible. La réciprocité qu'il exige de ses amis concerne aussi bien l'affection que les services. Et à aucun moment, Chapelain ne parle de l'échange de services comme d'une pratique intéressée qui mettrait en doute la sincérité des sentiments. Au contraire, se faire solliciter et solliciter l'autre est une preuve de confiance et d'amitié.

La coopération littéraire nécessite la confiance dans les intentions de l'autre et la foi dans les jugements mutuels. Elle donne parfois naissance à une fraternité amicale, affective et utilitaire à la fois.

\section{LETTERS OF JEAN CHAPELAIN: EVIDENCE OF HONEST AND USEFUL FRIENDSHIP BETWEEN MEN OF LETTERS}

Summary

Jean Chapelain corresponded with the most well-known writers, intellectuals and men of power. He was an authority in literature, a critic and theoretician, he advised Colbert but, at the same time, he was an object of mockery, a person in need of support from his friends. The article describes Chapelain's relations with friends where the aspect of usefulness, granting favours, reading and reviewing one another's works plays an important role in developing honest friendship.

Key words: friendship, Jean Chapelain, Guez de Balzac, Nicolas Heinsius, Gilles Ménage, sincere, useful Ménage, sincere, useful. 